\title{
Accuracy and Clinical Implications of Cincinnati Pre-Hospital Stroke Scale and Los Angeles Pre-Hospital Stroke Scale Use by Emergency Medical Services: A State Level Retrospective Study
} \author{
Souvik Sen ${ }^{1,5}$ \\ ${ }^{1}$ Department of Neurology, Palmetto Health Hospital, USA \\ ${ }^{2}$ Department of Epidemiology and Biostatistics, University of South Carolina, USA \\ ${ }^{3}$ Division of Chronic Disease Epidemiology, South Carolina Department of Health and Environmental Control, USA \\ ${ }^{4}$ Division of Emergency Medicine, Medical University of South Carolina, USA \\ ${ }^{5}$ School of Medicine, University of South Carolina, USA \\ ${ }^{6}$ Revenue and Fiscal Affairs, Office Health and Demographics, USA
}

Tushar Trivedi ${ }^{1,2 *}$, Khosrow Heidari ${ }^{3}$, Edward C Jauch ${ }^{4}$, Sonal Mehta ${ }^{1,5}$, Swamy Venkatesh ${ }^{1,5}$, Amanda Cotter ${ }^{1}$, Christopher Finney $^{6}$ and

\begin{abstract}
Background: The Cincinnati Pre-hospital Stroke Scale (CPSS) and Los Angeles Prehospital Stroke Scale (LAPSS) are widely used by EMS for screening of potential stroke cases.
\end{abstract}

Objectives: The objectives of this study were to assess the accuracy of CPSS and LAPSS when used by EMS in the field and evaluate the impact of their utilization on stroke treatment.

Methods: For the years 2010-2013, a state-level database was created linking South Carolina EMS data with hospital discharge records. For each scale, we calculated the sensitivity, specificity, positive and negative predictive value, comparing screening results with discharge diagnoses for stroke. Additionally, we evaluated the impact of early stroke identification by EMS using stroke scale, on treatment with IV-tPA.

Results: For all EMS transported cases where CPSS or LAPSS was used ( $n=101,442), 6,757$ cases had a confirmed stroke diagnosis. CPSS demonstrated sensitivity of $59 \%$,and specificity of $96 \%$. The sensitivity and specificity for LAPSS were $26 \%$ and $84 \%$, respectively. Positive and negative predictive values for CPSS were $45 \%$ and $98 \%$, and $27 \%$ and $83 \%$ for LAPSS, respectively. Rates of IV-tPA administration were approximately 5 times higher for those correctly identified using CPSS, and approximately twice higher for cases correctly identified using LAPSS.

Conclusion: Early identification of stroke cases using CPSS or LAPSS can have a significant impact on the rate of treatment of ischemic stroke with IV-tPA. We report a modest accuracy of these two stroke scales in correctly identifying stroke cases in the field, with CPSS leading to a higher rate of IV-tPA use compared with LAPSS.

Keywords: Emergency Medical Services (EMS); Cincinnati Prehospital Stroke Scale (CPSS); Los Angeles Prehospital Stroke Scale (LAPSS); Acute Ischemic Stroke; IV-tPA

\section{Introduction}

Stroke is the fifth leading cause of mortality in the United States (US) [1]. Timely arrival to a hospital facility and rapid evaluation are very critical in the management of acute stroke, leading to higher rates of treatment and improved clinical outcomes [2-4]. One of the most effective treatments for ischemic stroke, thrombolytic therapy with intravenous tissue plasminogen activator (IV tPA), has a narrow therapeutic window of just 4.5 hours from symptom onset and is more effective the sooner it is administered [5,6]. However, only $3 \%$ to $8 \%$ percent of patients with ischemic stroke receive tPA $[7,8]$, largely due to most stroke cases failing to arrive at the Emergency Department (ED) within the first several hours from symptom onset. This delay to presentation has been attributed to delays in self-recognition of stroke symptoms, delays in seeking medical attention by a stroke patient, and lack of 911 use producing delays in transporting the patient to a hospital capable of handling acute stroke cases [9-12]. The mode of transportation, particularly when Emergency Medical Services (EMS) are used, can have a positive impact in the care of acute stroke cases through early identification, expedited transport, prehospital notification, and EMS triage, thus allowing more timely evaluation and delivery of treatment [13-15]. These findings highlight the impact of accurate identification of stroke symptoms by EMS personnel using available screening tools, critical to guiding transportation of the cases to appropriate facilities in a timely manner.
The American Stroke Association recommends the use of screening tools such as Cincinnati Prehospital Stroke Scale (CPSS) or the Los Angeles Prehospital Stroke Screen (LAPSS) by EMS.16 The CPSS is based on physical examination only, and evaluates facial droop, speech abnormality and arm weakness [16]. Previous studies of the CPSS have reported sensitivity and specificity of $66 \%$ and $87 \%$ in ED [16], and $71 \%$ and $52 \%$ when used by paramedic's infield after interactive training [17]. LAPSS along with physical examination for facial smile or grimace, hand grip and arm strength also require that the provider rules out alternative possible causes of altered consciousness (e.g. hypoglycemia) [18]. Kidwell et al. reported sensitivity of $91 \%$ and a specificity of $97 \%$ for the use of LAPSS [18]. Previous studies that looked at the sensitivity and specificity of stroke scales were confined to EMS in small geographical locations, had a small sample size, and included paramedic training as a part of the research study. The

*Corresponding author: Tushar Trivedi, Department of Neurology, Palmetto Health Hospital, 8 Medical Park, Columbia, South Carolina 29201, USA, Tel: 347-302-5873; E-mail: tushar.trivedi@gmail.com

Received August 09, 2018; Accepted October 09, 2018; Published October 12, 2018

Citation: Trivedi T, Heidari K, Jauch E, Mehta S, Venkatesh S, et al. (2018) Accuracy and Clinical Implications of Cincinnati Pre-Hospital Stroke Scale and Los Angeles Pre-Hospital Stroke Scale Use by Emergency Medical Services: A State Level Retrospective Study. J Neurol Disord 6: 392. doi:10.4172/23296895.1000392

Copyright: ( $) 2018$ Trivedi T, et al. This is an open-access article distributed under the terms of the Creative Commons Attribution License, which permits unrestricted use, distribution, and reproduction in any medium, provided the original author and source are credited. 
Citation: Trivedi T, Heidari K, Jauch E, Mehta S, Venkatesh S, et al. (2018) Accuracy and Clinical Implications of Cincinnati Pre-Hospital Stroke Scale and Los Angeles Pre-Hospital Stroke Scale Use by Emergency Medical Services: A State Level Retrospective Study. J Neurol Disord 6: 392. doi:10.4172/2329-6895.1000392

Page 2 of 4

objective of this study is to perform a population-based field study to assess the accuracy of CPSS and LAPSS use by EMS. We also evaluated the potential impact of early identification of ischemic stroke by CPSS and LAPSS during EMS transport on treatment with IV tPA. To our knowledge this is the first population-based study to assess the accuracy of stroke scales performed on a state-wide database. We hypothesized the large sample and population-based approach would reflect a "real life" picture of the validity of prehospital stroke scales when used by the EMS in the field.

\section{Material and Methods}

A population-based study was conducted using the statewide EMS database linked with all South Carolina (SC) hospital discharge records, for the calendar years 2010-2013. The EMS data were obtained from the Division of EMS and Trauma at the Department of Health and Environmental Control and were linked to the hospitalization records housed at the Health and Demographics Division, SC Revenue Finance Affairs Department. These two datasets were linked based on a matching algorithm that includes social security number, first name, middle initial, last name, date of birth, race, and gender. The algorithm accounted for misspelling, name changes, transposed digits in the social security number and slight differences in the date of birth and was found to have an accuracy greater than $99 \%$ upon validation. SC EMS uses standard codes developed by National EMS Information System Initiative (NEMSIS) which has also served to provide technical assistance for the development and implementation of the EMS dataset. In the field, EMS personnel enter data from each encounter into a digital tool which has a preset of a questionnaire. For any suspected stroke case, where either CPSS or LAPSS is utilized, results can be recorded as a) positive; b) negative; or c) non-conclusive. However, none of the individual elements of either stroke scales are presently captured.

Of all the cases transported by SC EMS between 2010-2013, we identified 101,442 records where use of pre-hospital stroke scale was recorded by the paramedics (99,849 for CPSS and 1,593 with LAPSS). From this sample, we excluded 12,147 cases $(\sim 12.2 \%)$ where the results of pre-hospital stroke scale use were "non-conclusive". Our final study sample consisted of 89,295 cases with a conclusive (i.e., positive or negative) pre-stroke screen finding. This sample was linked with the

\begin{tabular}{|c|c|c|}
\hline Variables & Non-EMS Users & EMS Users \\
\hline Total N (\%) & $38,569(67.9 \%)$ & $18,194(32.1 \%)$ \\
\hline \multicolumn{3}{|l|}{ Age (Years) } \\
\hline$<30$ & $1.0 \%$ & $1.0 \%$ \\
\hline $30-60$ & $28.1 \%$ & $31.2 \%$ \\
\hline$>60$ & $70.9 \%$ & $67.7 \%$ \\
\hline \multicolumn{3}{|l|}{ Gender } \\
\hline Female & $52.9 \%$ & $50.9 \%$ \\
\hline Male & $47.1 \%$ & $49.1 \%$ \\
\hline \multicolumn{3}{|l|}{\begin{tabular}{|l|} 
Race $^{\dagger}$ \\
\end{tabular}} \\
\hline White & $70.6 \%$ & $61.5 \%$ \\
\hline African-American & $26.9 \%$ & $36.0 \%$ \\
\hline Others & $2.5 \%$ & $2.5 \%$ \\
\hline \multicolumn{3}{|l|}{ Insurance status } \\
\hline $\mathrm{HMO} /$ Private & $22.8 \%$ & $19.5 \%$ \\
\hline Medicare & $5.2 \%$ & $7.8 \%$ \\
\hline Medicaid & $61.8 \%$ & $60.9 \%$ \\
\hline Self-paid / Others & $10.2 \%$ & $11.7 \%$ \\
\hline \multicolumn{3}{|l|}{ Stroke type ${ }^{\dagger}$} \\
\hline Hemorrhagic & $9.2 \%$ & $19.3 \%$ \\
\hline Ischemic & $57.0 \%$ & $52.0 \%$ \\
\hline TIA & $27.6 \%$ & $22.4 \%$ \\
\hline Non-specific & $6.2 \%$ & $6.3 \%$ \\
\hline \multicolumn{3}{|l|}{ Hospital type } \\
\hline Certified Primary Stroke Center ${ }^{*}$ & $51.3 \%$ & $50.3 \%$ \\
\hline Non-Certified & $48.7 \%$ & $49.7 \%$ \\
\hline tPA rates (Ischemic stroke cases) ${ }^{\dagger}$ & $3.2 \%$ & $9.1 \%$ \\
\hline
\end{tabular}

Table 1: Selected characteristics of all stroke patients admitted in SC hospital by mode of transportation during 2010-2013.

\begin{tabular}{|c|c|c|c|c|c|}
\hline \multicolumn{3}{|c|}{ CPSS (n=99849) } & \multicolumn{3}{c|}{ LAPSS (n=1593) } \\
\hline TP & FP & PPV (95\% Cl) & TP & FP & PPV (95\% Cl) \\
\hline 2909 & 3639 & $0.44(0.43-0.45)$ & 67 & 179 & $0.27(0.22-0.33)$ \\
\hline FN & TN & NPV (95\% Cl) & FN & TN & NPV (95\% Cl) \\
\hline 2025 & 79378 & $0.97(0.97-0.98)$ & 191 & 907 & $0.82(0.80-0.84)$ \\
\hline Sens & Spec & Accuracy & Sens & Spec & Accuracy \\
\hline $0.58(0.57-0.60)$ & $0.95(0.95-0.96)$ & $0.93(0.92-0.94)$ & $0.26(0.20-0.31)$ & $0.83(0.81-0.85)$ & $0.72(0.68-0.75)$ \\
\hline
\end{tabular}

Abbreviations: TP-True Positive; FP- False Positive; TN- True Negative; FN: False Negative; PPV: Positive Predictive Value; NPV-Negative Predictive Value; SensSensitivity; Spec- Specificity.

Table 2: Accuracy of CPSS and LAPSS when compared with the hospital discharge diagnosis, South Carolina during $2010-2013$. 
Citation: Trivedi T, Heidari K, Jauch E, Mehta S, Venkatesh S, et al. (2018) Accuracy and Clinical Implications of Cincinnati Pre-Hospital Stroke Scale and Los Angeles Pre-Hospital Stroke Scale Use by Emergency Medical Services: A State Level Retrospective Study. J Neurol Disord 6: 392. doi:10.4172/2329-6895.1000392

Page 3 of 4

statewide hospitalization records based on the matching algorithm discussed above. In this linked dataset we compared the results from pre-hospital stroke scale use by the EMS with the primary hospital discharge diagnoses for Cerebrovascular Accident (CVA). Hospital discharge diagnosis for CVA was determined using ICD-9 codes (430.0-438.9) and was considered as the gold standard for reference. CVA included cases of ischemic stroke, transient ischemic attack, intracerebral hemorrhage, subarachnoid hemorrhage, and another nonspecific stroke. We calculated the Sensitivity (Sens), Specificity (Spec), Positive Predictive Value (PPV), Negative Predictive Value (NPV), and Accuracy of CPSS and LAPSS. Additionally, we evaluated the impact of stroke identification using these scales by the EMS on the rates of IV tPA treatment in ischemic stroke. Use of tPA was determined using ICD-9 procedure code for injection or infusion of thrombolytic agent (99.10). Further, we also produced descriptive statistics for all stroke patients (a total of 56,763 cases) admitted to SC hospitals over the study period, by their mode of transportation (EMS $v s$. Non-EMS users). All statistical analyses were performed on SAS 9.4 (SAS Institute, Cary, NC). Chi-square test of independence was used to assess the significance of the observed differences between EMS $v s$. Non-EMS transportation.

\section{Results}

Patient and hospital level characteristics for all stroke patients admitted in SC hospital system from 2010 to 2013 are summarized in Table 1. Of the total 56,763 admitted stroke cases only $32 \%$ utilized EMS services. The proportion of patients with hemorrhagic stroke was twice as high among EMS users as compared to Non-EMS users, likely owing to the stroke severity (19.3\% in EMS vs. 9.1\% in Non-EMS users). There were no major differences by age, gender, insurance, or the type of destination hospital (certified primary stroke centers $v s$. non-certified) among EMS and Non-EMS users. However, the proportion of AfricanAmericans was slightly higher in EMS users as compared to Non-EMS users. Among ischemic stroke cases, rates of IV tPA administration was 3 times higher for cases transported by EMS as compared to Non-EMS users $(9.1 \%$ vs. $3.2 \%$, p-value $<0.01)$.

Of all the cases that utilized EMS services in SC over the study period, use of a stroke scale with conclusive results was recorded for 89,295 cases (CPSS was used in $98.5 \%$ of these cases). Among these cases, 6,757 had a diagnosis of stroke or TIA as confirmed by hospital discharge records. Paramedics' use of CPSS demonstrated Sens of $58.9 \%$, Spec of $95.6 \%$, PPV of $44.4 \%$ and NPV of $97.5 \%$ (Table 2). For LAPSS, Sens of $25.9 \%$, Spec of $83.5 \%$, PPV of $27.2 \%$ and NPV of $82.6 \%$ were observed. Overall accuracy for CPSS and LAPSS were $93 \%$ and $72 \%$, respectively (Table 2). For the ischemic stroke cases where CPSS was used for screening, rates of IV tPA administration were about 5

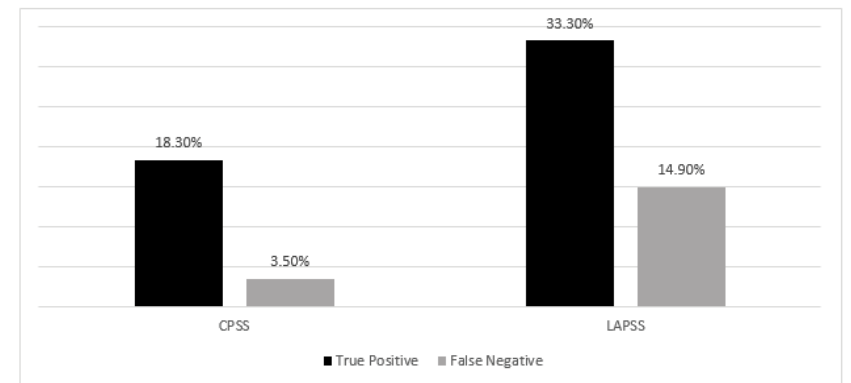

Figure 1: Rates of IV IPA administration for ischemic stroke cases by the type and results of stroke scale used by the EMS times higher for those correctly identified as a stroke (i.e., true positive) than those falsely identified as a non-stroke (i.e., false negative) $(18.3 \%$ for true positive $v s .3 .5 \%$ for false negative, $\mathrm{p}<0.01$ ). For LAPSS, rates for IV tPA administration were 2 times higher in true positives than false negatives $(33.3 \%$ vs. $14.9 \%, \mathrm{p}<0.01)$ (Figure 1).

\section{Discussion}

Studies from different parts of the US have shown EMS utilization of up to $50 \%$ by stroke cases $[12,19,20]$. In contrast, of all the hospitalized stroke cases in SC in the years 2010-2013, roughly one-third utilized EMS as their mode of transport to arrive at hospital. It is relevant in the context that our findings indicate that the utilization of EMS is associated with higher rates of IV tPA administration, approximately 3 times higher than non-EMS users. Additionally, it is worth noting among all the EMS transported cases identified as "suspected stroke" by the paramedics, only $54 \%$ were transported to a certified primary stroke center (PSC) (data not shown). Of the cases not transported to a stroke center many may have been too far from a PSC or some may even have been transported to a tele stroke center, but the fact that currently there are no EMS triage policies in SC for the transport of a suspected stroke patient to a nearest stroke center likely played a role.

The primary finding of this study is that the use of pre-hospital stroke scales by the paramedics in the field has low accuracy in identifying acute stroke, with only $26 \%$ and $58 \%$ of stroke cases were correctly identified by LAPPS and CPSS respectively. Further, we found for ischemic stroke cases where the prehospital stroke scales correctly identified acute stroke, the rate of tPA administration was about 5 times higher with CPSS use and 2 times higher with LAPSS as compared to the false negatives.

Prior studies have shown varying levels of accuracy of prehospital stroke recognition by EMS using these stroke scales. In comparison to our study, where we found a Sens of 59\% and Spec of 96\% for CPSS, an EMS field study done by Frendl et al. reported modestly higher Sens of $71 \%$ and lower Spec of $52 \%$ for CPSS [17]. However, their study had a much smaller sample size $(n=154)$ and the EMS were confined to a smaller geographical area (Durham county, North Carolina). During the validation stage of CPSS, Kothari et al. reported Sens of $59 \%$ and Spec of $88 \%$ which were like the values reported in our study (17). A study done by Kidwell et al. for the validation of LAPSS reported Sens of $91 \%$ and Spec of $97 \%$, which is much higher than our observed values of $26 \%$ and $84 \%$ respectively [18]. It is noteworthy that the paramedics were trained and certified for the use of LAPSS as a part of their study, and the study data was confined to EMS at a single university hospital, with a small sample size $(n=206)$ [18]. It is difficult to explain the rationale for the observed differences in the Sens when compared to our study based on these reasons alone; however, lack of structured paramedic's training for LAPSS utilization may have played a significant role.

\section{Limitations}

Our study has a few limitations. First, since we relied on the EMS documentation as an indication of performance of stroke screening measures, our results may be biased if the documentation were not accurate. At this time point, we do not have the means to validate the accuracy of data entry by the EMS, however considering the differences observed, it would be worth examining in future. Second, false negative pre-hospital stroke screen findings may have been more common among cases that had milder stroke symptoms, cases who might have called EMS late, and because of the time lapse were not eligible for treatment with IV tPA. Thus, our study finding suggesting high IV tPA 
Citation: Trivedi T, Heidari K, Jauch E, Mehta S, Venkatesh S, et al. (2018) Accuracy and Clinical Implications of Cincinnati Pre-Hospital Stroke Scale and Los Angeles Pre-Hospital Stroke Scale Use by Emergency Medical Services: A State Level Retrospective Study. J Neurol Disord 6: 392. doi:10.4172/2329-6895.1000392

Page 4 of 4

rate among cases correctly identified as an acute stroke by EMS (using pre-hospital stroke screen) may somewhat be an overestimation. At this time, we do not have relevant information available in the dataset to adjust for the severity of stroke, which would perhaps give a more unbiased estimate. Third, we excluded approximately $12 \%$ of all EMS transported cases with non-conclusive pre-hospital stroke screening findings, which were more likely with LAPSS (16\%) than CPSS (11.5\%). Non-conclusive results can possibly be associated with stroke severity, as severe stroke cases would less likely be able to follow screening instructions yielding high non-conclusive results. If this was the case, it would introduce a selection bias from differential selection of screening population (based on stroke severity) for each stroke scale. However, the magnitude of this bias would be small considering the small difference in the proportion of non-conclusive cases for the two scales. Having said that, we should keep in mind the sample size for the study population are quite different for CPSS $(n=99,849)$ and LAPSS $(n=1,593)$ and this could be reflected in the results. Finally, our data represents a pooled data set of different EMS agencies throughout the state, and we lack information to compare the capabilities and resources of EMS agencies that are known to vary substantially by region [21].

\section{Conclusion}

The results from our study suggest a modest accuracy of CPSS and LAPSS in identifying stroke cases when used by paramedics in the field. Nonetheless, early identification of "true stroke" cases by EMS had a positive impact on treatment, where accurate prehospital stroke identification using stroke scale was associated with higher rates of IVtPA administration. Considering early identification of stroke cases by EMS can have a have a significant impact on timely delivery of treatment, further research is warranted to improve the accuracy of prehospital stroke scales, either by extensive training and ongoing performance improvement programs or by finding alternative screening methods that can be efficiently utilized by EMS. Furthermore, programs and policies should be put in place to address the underutilization of EMS by stroke cases in South Carolina.

\section{References}

1. Mozaffarian D, Benjamin EJ, Go AS, Arnett DK, Blaha MJ, et al. (2015) Heart disease and stroke statistics-2015 update: a report from the American Heart Association. Circulation 131: e29.

2. Sheppard JP, Mellor RM, Greenfield S, Mant J, Quinn T, et al. (2013) The association between prehospital care and in-hospital treatment decisions in acute stroke: A cohort study. Emerg Med J emermed 32: 93-99.

3. Frankel M, Hinchey J, Schwamm LH, Croft JB (2007) Prehospital and hospital delays after stroke onset-United States, 2005-2006. Morbidity and mortality weekly report 56: 474 .

4. Fonarow GC, Smith EE, Saver JL, Reeves MJ, Hernandez AF, et al. (2011) Improving door-to-needle times in acute ischemic stroke the design and rationale for the American Heart Association/American Stroke Association's target: stroke initiative. Stroke 42: 2983-2989.

5. Marler JR, Tilley BC, Lu M, Brott TG, Lyden PC, et al. (2000) Early stroke treatment associated with better outcome The NINDS rt-PA Stroke Study. Neurology 55: 1649-1655.

6. Jauch EC, Saver JL, Adams HP Jr, Bruno A, Connors JJ, et al. (2013) Guidelines for the early management of patients with acute ischemic stroke a guideline for healthcare professionals from the American Heart Association/ American Stroke Association. Stroke 44: 870-947.

7. Alberts MJ, Latchaw RE, Selman WR, Shephard T, Hadley MN, et al. (2005) Recommendations for Comprehensive Stroke Centers a Consensus Statement From the Brain Attack Coalition. Stroke 36: 1597-1616.

8. Reeves MJ, Arora S, Broderick JP, Frankel M, Heinrich JP, et al. (2005) Acute stroke care in the US Results from 4 Pilot Prototypes of the Paul Coverdell National Acute Stroke Registry. Stroke 36: 1232-1240.

9. Smith MA, Doliszny KM, Shahar E, McGovern PG, Arnett DK, et al. (1998) Delayed hospital arrival for acute stroke: the Minnesota Stroke Survey. Ann Intern Med 129: 190-196.

10. Rosamond WD, Gorton RA, Hinn AR, Hohenhaus SM, Morris DL (1998) Rapid response to stroke symptoms: The Delay in Accessing Stroke Healthcare (DASH) study. Acad Emerg Med 5: 45-51.

11. Morris DL, Rosamond W, Madden K, Schultz C, Hamilton S (2000) Pre-hospital and emergency department delays after acute stroke the Genentech Stroke Presentation Survey. Stroke 31: 2585-2590.

12. Schroeder EB, Rosamond WD, Morris DL, Evenson KR, Hinn AR (2000) Determinants of use of Emergency medical services in a population with stroke symptoms the second delay in accessing stroke healthcare (DASH II) Study. Stroke 31: 2591-2596.

13. Lin CB, Peterson ED, Smith EE, Saver JL, Liang L, et al. (2012) Emergency medical service hospital prenotification is associated with improved evaluation and treatment of acute ischemic stroke. Circ Cardiovasc Qual Outcomes 5: 514-522.

14. Patel MD, Rose KM, O'Brien EC, Rosamond WD (2011) Prehospital notification by emergency medical services reduces delays in stroke evaluation findings from the North Carolina stroke care collaborative. Stroke 42: 2263-2268.

15. Schwamm LH, Pancioli A, Acker JE 3rd, Goldstein LB, Zorowitz RD, et al (2005) Recommendations for the establishment of stroke systems of care recommendations from the American Stroke Association's task force on the development of stroke systems. Stroke 36: 690-703.

16. Kothari RU, Pancioli A, Liu T, Brott T, Broderick J (1999) Cincinnati prehospital stroke scale: reproducibility and validity. Ann Emerg Med 33: 373-378.

17. Frendl DM, Strauss DG, Underhill BK, Goldstein LB (2009) Lack of impact of paramedic training and use of the cincinnati prehospital stroke scale on stroke patient identification and on-scene time. Stroke 40: 754-756.

18. Kidwell CS, Starkman S, Eckstein M, Weems K, Saver JL (2000) Identifying stroke in the field prospective validation of the Los Angeles Prehospital Stroke Screen (LAPSS). Stroke 31: 71-76.

19. Barsan WG, Brott TG, Broderick JP, Haley EC, Levy DE, et al. (1993) Time of hospital presentation in patients with acute stroke. Arch Intern Med 153: 2558-2561.

20. Adeoye O, Lindsell C, Broderick J, Alwell K, Jauch E, et al. (2007) Emergency Medical Services Utilization by Stroke Patients: A Population-based Study. Am J Emerg Med 14: S159-S159.

21. Brice JH, Evenson KR, Lellis JC, Rosamond WD, Aytur SA, et al. (2008) Emergency Medical Services Education, Community Outreach, and Protocols for Stroke and Chest Pain in North Carolina. Prehosp Emerg Care 12: 366-371. 